\title{
Lemmel's Syndrome: A Rare Cause of Obstructive Jaundice Secondary to Periampullary Diverticulum
}

\author{
Babar Ahmad Khan, Sania Hanif Khan, Anuj Sharma \\ Department Of Internal Medicine, SUNY Upstate Medical University, Syracuse, NY, USA
}

Received: 25/04/2017

Accepted: 27/04/2017

Published: 05/05/2017

How to cite this article: Khan BA, Khan SH, Sharma A. Lemmel's syndrome: a rare cause of obstructive jaundice secondary to periampullary diverticulum. EJCRIM 2017;4: doi:10.12890/2017_000632.

Conflicts of Interests: The Authors declare that there are no competing interests.

This article is licensed under a Commons Attribution Non-Commercial 4.0 License

\section{ABSTRACT}

In 1934 Lemmel was the first to report the presence of juxtapapillary diverticula and hepatocholangiopancreatic disease, excluding cholelithiasis. Obstructive jaundice caused by periampullary duodenal diverticulum (PAD) in absence of choledocholithiasis or tumor is known as Lemmel syndrome. A patient with an extraluminal duodenal diverticulum presenting with obstructive jaundice and pancreatitis is presented in this case. Although initially managed conservatively, the patient had recurrence of symptoms after 2 months but then had successful surgical resection of the duodenal diverticulum.

\section{LEARNING POINTS}

- Lemmel's syndrome should be considered in patients with pancreaticobiliary disease in the absence of tumors, stricture, or cholelithiasis.

- Non-invasive imaging studies should be considered first but endoscopic retrograde cholangiopancreatography (ERCP) remains the diagnostic method of choice.

- Surgical resection (diverticulectomy), endoscopic sphincterotomy, and papillary balloon dilatation are treatment options when conservative management fails.

\section{KEYWORDS}

Lemmel's syndrome, juxtapapillary diverticula, periampullary duodenal diverticulum (PAD), choledocholithiasis, cholelithiasis, ERCP, MRCP, ampulla of Vater, pancreaticobiliary.

\section{INTRODUCTION}

Lemmel's syndrome is defined as obstructive jaundice caused by periampullary duodenal diverticulum (PAD) in absence of choledocholithiasis or tumor. A duodenal diverticulum is a sac-like out-pouching of the duodenal mucosa. Duodenal diverticula are mostly asymptomatic and found incidentally in up to $22 \%$ of the population, out of which less than $10 \%$ are symptomatic.

These are categorized into extramural and intramural diverticula, the majority of which are extra-luminal. Extra-luminal duodenal diverticulum (EDD) is a herniation acquired from a defect in the bowel wall due to entrance of vessels. Intramural duodenal diverticulum (IDD) is a rare congenital anomaly resulting from incomplete canalization of the lumen. Clinicians should always consider diverticulum as a possible cause of pancreatitis and obstructive jaundice ${ }^{[1-5]}$. 


\section{CASE PRESENTATION}

A rare case of a 69-year-old male is reported. He presented with abrupt onset of sharp epigastric pain, 10/10 in intensity, radiating to his back, worse in supine position and relieved by bending forward. The symptoms started 2-3 hours after a family dinner during the evening prior to admission. He denied any history of alcohol abuse or cholelithiasis. Murphy's sign was equivocal on exam. Triglycerides on admission were $151 \mathrm{mg} / \mathrm{dL}$, infectious hepatitis and autoimmune pancreatitis work-up (ig-G subclasses) were negative. However, lipase was elevated at $5168 \mathrm{IU} / \mathrm{L}$ so the patient was made NPO and started on intravenous fluids. Total bilirubin peaked at $2.9 \mathrm{mg} / \mathrm{dL}$ and direct bilirubin at $1.4 \mathrm{mg} / \mathrm{dL}$ on day-3. Transaminases were grossly normal on presentation but later peaked with ALT of 50 IU/L and AST of 59 IU/L on day-3. Alkaline phosphatase remained borderline elevated (156 IU/L). CT scan demonstrated diffuse stranding around the pancreatic head and adjacent duodenum (Fig.1).

MRCP confirmed a $1.1 \mathrm{~cm}$ ovoid cystic lesion adjacent to the descending duodenum consistent with a duodenal diverticulum (Fig. 2).

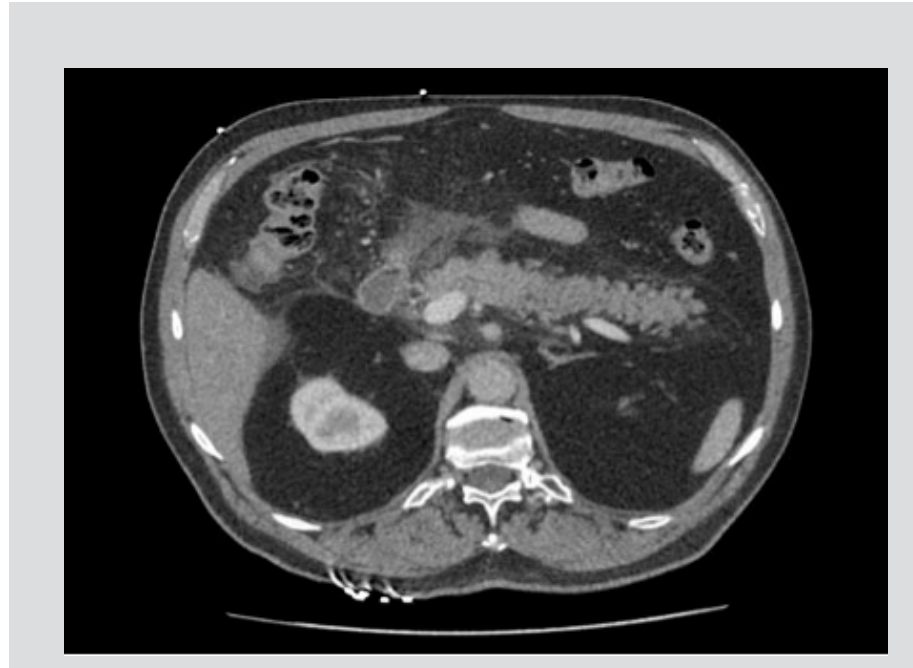

Figure 1. CT scan demonstrating diffuse stranding around the pancreatic head and adjacent duodenum

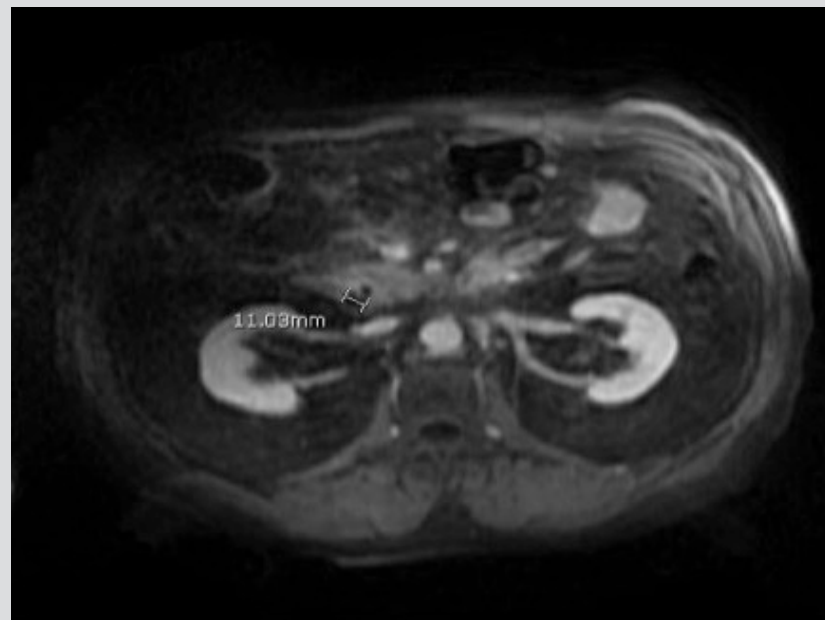

Figure 2. MRCP showing a $1.1 \mathrm{~cm}$ ovoid cystic lesion adjacent to the descending duodenum consistent with a duodenal diverticulum

The pancreatic duct was also moderately dilated but it was decided to proceed with medical management since LFTs started trending down and were normal by day-6. Mechanical obstruction caused by extrinsic compression of the duodenal diverticulum was considered to be responsible for the obstructive jaundice and pancreatitis. Eight weeks later the patient had a second episode of obstructive jaundice and underwent surgical resection of the duodenal diverticulum, not only confirming the diagnosis but also resulting in resolution of his symptoms.

\section{DISCUSSION}

Most of the duodenal diverticula are true, comprising only the mucosal outpouching without any muscle layer. They are primarily solitary diverticula and up to $75 \%$ arise out of the second part of the duodenum in proximity to the ampulla of Vater ${ }^{[3]}$. Extra-luminal diverticulum, found within a radius of $3 \mathrm{~cm}$ from the ampulla of Vater, is known as periampullary diverticulum (PAD) ${ }^{[2,3]}$. Their prevalence has been reported as $0.16 \%-22 \%$ depending on the sensitivity of the diagnostic method used. The majority of these diverticula are diagnosed incidentally and are asymptomatic but at times pancreaticobiliary and/or non-pancreaticobiliary complications may occur. Hemorrhage, fistula, or perforation and enterolith formation are the major non-pancreaticobiliary complications and their pathophysiology revolves around inflammation of the diverticulum whereas the pancreaticobiliary complications include gall-bladder and bile-duct stones, obstructive jaundice, cholangitis, as well as acute and chronic pancreatitis. These complications are primarily due to the extrinsic compression of Oddi dysfunction by the diverticulum, sphincter, and regurge of intestinal contents in biliary ducts resulting in bacterial overgrowth ${ }^{[3-5]}$.

Prevalence of PAD has been reported to be as high as $65 \%$ in elderly population. Although mostly asymptomatic, it can present albeit rarely as obstructive jaundice in the absence of choledocholithiasis or tumor due to extrinsic compression of the CBD. 
This unusual presentation of obstructive jaundice secondary to PAD is often due to an impacted intra-diverticular enterolith and is known as Lemmel's syndrome ${ }^{[2]}$.

Diagnosing Lemmel's syndrome is often very challenging, so great awareness about Lemmel's syndrome in patients with periampullary diverticula is extremely important in avoiding delays in diagnosis and management of these patients. PADs appear as contrast filled outpouchings, originating from the medial aspect of the duodenum in barium study. CT can show either cystic or solid lesion on the medial wall of descending duodenum often mistaken for pancreatic pseudo-cyst, abscess, or cystic neoplasm. MRCP can be used to distinguish diverticulum from pseudocysts or cystic tumors of the pancreatic head. However, a side-viewing endoscope during ERCP is considered to be the gold-standard diagnostic test ${ }^{[2,3]}$.

Diverticulectomy remains the standard of care, however in patients where diverticulectomy is not possible or the patient is a high-risk surgical candidate, endoscopic sphincterotomy, papillary balloon dilatation and conservative medical management are alternate acceptable options ${ }^{[1,2]}$.

\section{REFERENCES}

1. Buse PE, Edmundowicz SA. Proximal common bile duct obstruction secondary to a periampullary duodenal diverticulum: successful treatment with endoscopic stenting. Gastrointest Endosc 1991;37:635-7.

2. Kang HS, Hyun JJ, Kim SY, Jung SW, Koo JS, Yim HJ, et al. Lemmel's syndrome, an unusual cause of abdominal pain and jaundice by impacted intradiverticular enterolith: case report. J Korean Med Sci 2014;29:874-8.

3. Karayiannakis AJ, Bolanaki H, Courcoutsakis N, Kouklakis G, Moustafa E, Prassopoulos P, et al. Common bile duct obstruction secondary to a periampullary diverticulum. Case Rep Gastroenterol 2012;6:523-9.

4. Rouet J, Gaujoux S, Ronot M, Palazzo M, Cauchy F, Vilgrain V, et al. Lemmel's syndrome as a rare cause of obstructive jaundice. Clin Res Hepatol Gastroenterol 2012;36:628-31.

5. Tan NC, Ibrahim S, Chen CM, Tay KH. Periampullary diverticulum causing biliary stricture and obstruction. Singapore Med J 2005;46:250-1. 